\title{
Neuropeptide Y Overexpression Using Recombinant Adenoassociated Viral Vectors
}

\author{
Francesco Noé,* Angelisa Frasca,* Claudia Balducci,* Mirjana Carli,* Gunther Sperk, ${ }^{\dagger}$ \\ Francesco Ferraguti, ${ }^{\dagger}$ Asla Pitkänen, ${ }^{\ddagger}{ }^{\ddagger}$ Ross Bland, ${ }^{\S \Psi}$ Helen Fitzsimons, ${ }^{\S \nsubseteq}$ \\ Matthew During, ${ }^{\text {II }}$ and Annamaria Vezzani* \\ *Department of Neuroscience, Mario Negri Institute for Pharmacological Research, Milano, 20156 Italy, ${ }^{\dagger}$ Department of \\ Pharmacology, Medical University, A-6020 Innsbruck, Austria, ${ }^{\ddagger}$ Epilepsy Research Lab, A.I. Virtanen Institute for Molecular Science, \\ University of Kuopio, "Department of Neurology, Kuopio University Hospital, Kuopio, FI-70211 Finland, ${ }^{\S}$ Neurologix, Inc., Fort Lee, \\ New Jersey 07024, ${ }^{\mathbb{T}}$ Human Cancer Genetics Programme, The Ohio State University, Columbus, Ohio 43210
}

\begin{abstract}
Summary: Gene therapy may represent a promising alternative treatment of epileptic patients who are resistant to conventional anti-epileptic drugs. Among the various approaches for the application of gene therapy in the treatment of CNS disorders, recombinant adeno-associated viral (AAV) vectors have been most widely used. Preclinical studies using a selection of "therapeutic" genes injected into the rodent brain to correct the compromised balance between inhibitory and excitatory transmission in epilepsy, showed significant reduction of seizures and inhibition of epileptogenesis. In particular, transduction of neuropeptide genes, such as galanin and neuropeptide Y (NPY) in specific brain areas in experimental models of seizures re-
\end{abstract}

sulted in significant anticonvulsant effects. Recent findings showed a long-lasting NPY over-expression in the rat hippocampus by local application of recombinant AAV vectors associated with reduced generalization of seizures, delayed kindling epileptogenesis, and strong reduction of chronic spontaneous seizures. These results establish a proof-ofprinciple evidence of the efficacy of gene therapy as anticonvulsant treatment. Additional investigations are required to address safety concerns and possible side effects in more detail. Key Words: Adeno-associated viral vectors, anticonvulsant, gene therapy in epilepsy, neuropeptides, temporal lobe epilepsy.

\section{INTRODUCTION}

Epilepsy, the third most common neurological disease, is a chronic CNS disorder characterized by recurrent spontaneous seizures. Current antiepileptic drugs (AEDs) mainly target transmitter receptors and ion channels, ${ }^{1}$ and are effective in only 60 to $70 \%$ of individuals. Less than $10 \%$ of patients refractory to two or more AEDs are suitable for surgical resection of the epileptic focus as a final therapeutic option., ${ }^{2,3}$ Gene therapy techniques provide a realistic alternative to resective surgery by offering the possibility to express a "therapeutic" gene in the epileptic focus by avoiding tissue ablation. ${ }^{4}$ Neurotropic adeno-associated viral (AAV) vectors represent the tool of choice for gene delivery in experimental models of epi-

Address correspondence and reprint requests to: Annamaria Vezzani, $\mathrm{PhD}$, Department of Neuroscience, Mario Negri Institute for Pharmacological Research, Via G. La Masa 19, Milano, 20156 Italy. E-mail: vezzani@marionegri.it. lepsy. These vectors present many advantages since they can efficiently express single or multiple transgenes from a wide range of regulatory elements in neurons; they can be engineered at the capsid and promoter level to preferentially target specific populations of neurons in a controllable manner; very importantly they are nonpathogenic and appear not to affect normal brain physiology. ${ }^{5}$

There is a wide range of potential therapeutic genes that could inhibit neuronal hyperexcitability and the choice of the appropriate gene is one crucial aspect for a successful gene therapy approach. Among the transgenes expressed in CNS so far using AAV vectors there are: the constitutive NMDA receptor subunit (NR1), which was downregulated by AAV expression of a specific antisense transcript to reduce the number of functional NMDA receptors, thus impairing the glutamate-mediated excitatory transmission; ${ }^{6}$ the $\mathrm{GABA}_{\mathrm{A}}$ receptor alpha 1 subunit that was increased in granule cells using a seizure-activated promoter, and the neuropeptides, such as 
galanin and NPY. ${ }^{7-11}$ Using pharmacological approaches and transgenic rodents, galanin and NPY were previously shown to be endowed of powerful anticonvulsant activities in various experimental models of seizures and epileptogenesis. ${ }^{8}$

\section{NPY AS AN ENDOGENOUS ANTICONVULSANT PEPTIDE}

NPY overexpression in the hippocampus and temporal cortex has been described after experimentally induced seizures and in human epileptogenic tissue from temporal lobe epileptic patients. ${ }^{9,12,13}$ Changes in NPY expression are concomitant to modifications in its receptor subtypes, thus suggesting that NPY-mediated neurotransmission is altered in the epileptic tissue. A high density of NPY Y2 receptors is observed in the terminal field of mossy fibers and Y1 receptors are down-regulated in the molecular layer of the dentate gyrus in chronic epileptic specimens. ${ }^{14}$

Pharmacological and functional studies showed anticonvulsant actions of NPY in experimental models of seizures (see as follows), as well as in the epileptic human hippocampus. ${ }^{15}$
In experimental models of seizures, administration of NPY or Y2/Y5 receptor agonists results in strong anticonvulsant effects. ${ }^{9,13}$ Accordingly, mice with a deletion of the NPY gene or its receptor subtypes were more susceptible to seizures. ${ }^{16,17}$ The NPY-mediated inhibition of excitatory glutamatergic neurotransmission in the hippocampus is responsible for the NPY anticonvulsant effects. ${ }^{18,19}$ These findings indicate that increased NPY transmission in the hippocampus results in prominent anticonvulsant effects, therefore suggesting that AAVmediated peptide overexpression may represent an effective antiepileptic strategy.

\section{THE NPY GENE AS A THERAPEUTIC TARGET: STUDIES IN EXPERIMENTAL MODELS}

As a first approach to deliver and express the NPY gene in the rat brain, we used AAV vectors with different serotypes and targeted the hippocampus, the area where seizures originate in our experimental models. We showed that the efficiency of AAV vector to transduce hippocampal neurons varies, depending on the capsid
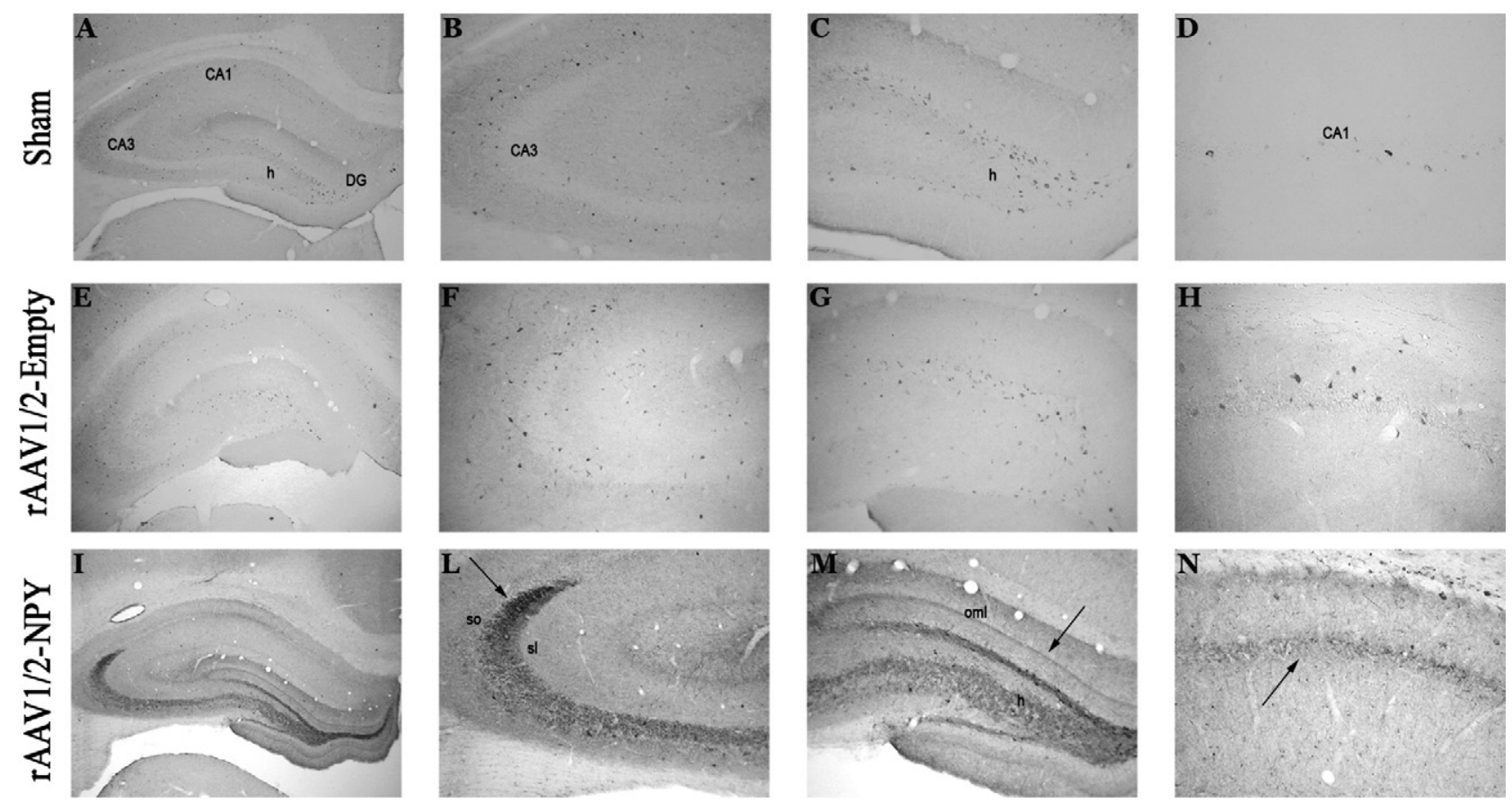

FIG. 1. Neuropeptide Y (NPY) overexpression in the hippocampus of rats injected with recombinent ( $r$ ) adeno-associated viral (AAV) 1/2 vector. Representative micrographs of the immunohistochemical section from injected dorsal rat hippocampus (4x), and magnification of CA3, hilus, and CA1 areas (10x) in Sham (rats injected with saline, A-D), AAV1/2-empty (rats injected with the vector lacking the transgene, E-H), and AAV1/2-NPY (rats injected with the vector expressing NPY under the control of the CBA promoter, I-N). Naive rats were injected with the vectors ${ }^{20}$ and killed 4 weeks thereafter. NPY staining was similarly present in interneurons in Sham and AAV1/2-CBA-NPY rats, in AAV1/2-NPY increase staining was prominently observed in the terminal field of mossy fibers (arrow in L), in the outer molecular layer (oml) of the dentate gyrus (DG, arrow in M), and in CA1 representing NPY in fibers (arrow in $\mathrm{N}$ ) (arrow in the outer molecular layer in $\mathrm{M}$ depicts the region where hilar interneurons project). $\mathrm{h}=\mathrm{hilus}$; $\mathrm{sl}=$ strata orient; so $=$ lacunosum. 
genes. Thus, serotype 2 AAV vector increased NPY expression in hilar interneurons only, ${ }^{20}$ a population of cells that normally express NPY in physiological conditions, whereas the chimeric serotype $1 / 2$ vector caused more widespread expression, including ectopic expression in mossy fibers and in pyramidal cells and the subiculum. ${ }^{20}$ Figure 1 shows the pattern of AAV1/2-mediated NPY overexpression in the hippocampus driven by the cytomegalovirus-chicken- $\beta$-actin (CBA) promoter. This pattern is very similar to the one observed using the neuron-specific-enolase promoter. $^{20}$ Our unpublished data show that AAV-mediated NPY overexpression in the hippocampus lasts for at least 6 months in rats. Histological evaluation of brain tissue close to the AAV vector injection site did not show neuronal cell loss (FIG. 2), or microglia or astroglia activation any different from the changes related to the mechanical damage in- duced by the injection needle. Notably, inflammation was absent in vector-injected tissue as assessed by measuring IL-1 $\beta$ immunostaining (FIG. 2).

Radioimmunoassay measurements demonstrated an average 10-fold increase in NPY levels induced by AAV1/2-CBA vector in the hippocampus. ${ }^{21}$ HPLC analysis showed that NPY encoded by the transfected neuronal cells is eluted with synthetic rat NPY, which indicates that transduced and endogenous NPY do not differ. AAV1/2 mediated NPY overexpression results in enhanced $\mathrm{KCl}$-induced release from hippocampal slices as compared to slices obtained from empty-vector-injected rats (control rats receiving a vector with a cassette lacking the transgene). Importantly, levels of NPY Y2, measured by ligand binding, in the hippocampus of AAV1/ 2-CBA-NPY injected rats were not affected by NPY overexpression, whereas $\mathrm{Y} 1$ receptors were reduced ${ }^{21}$
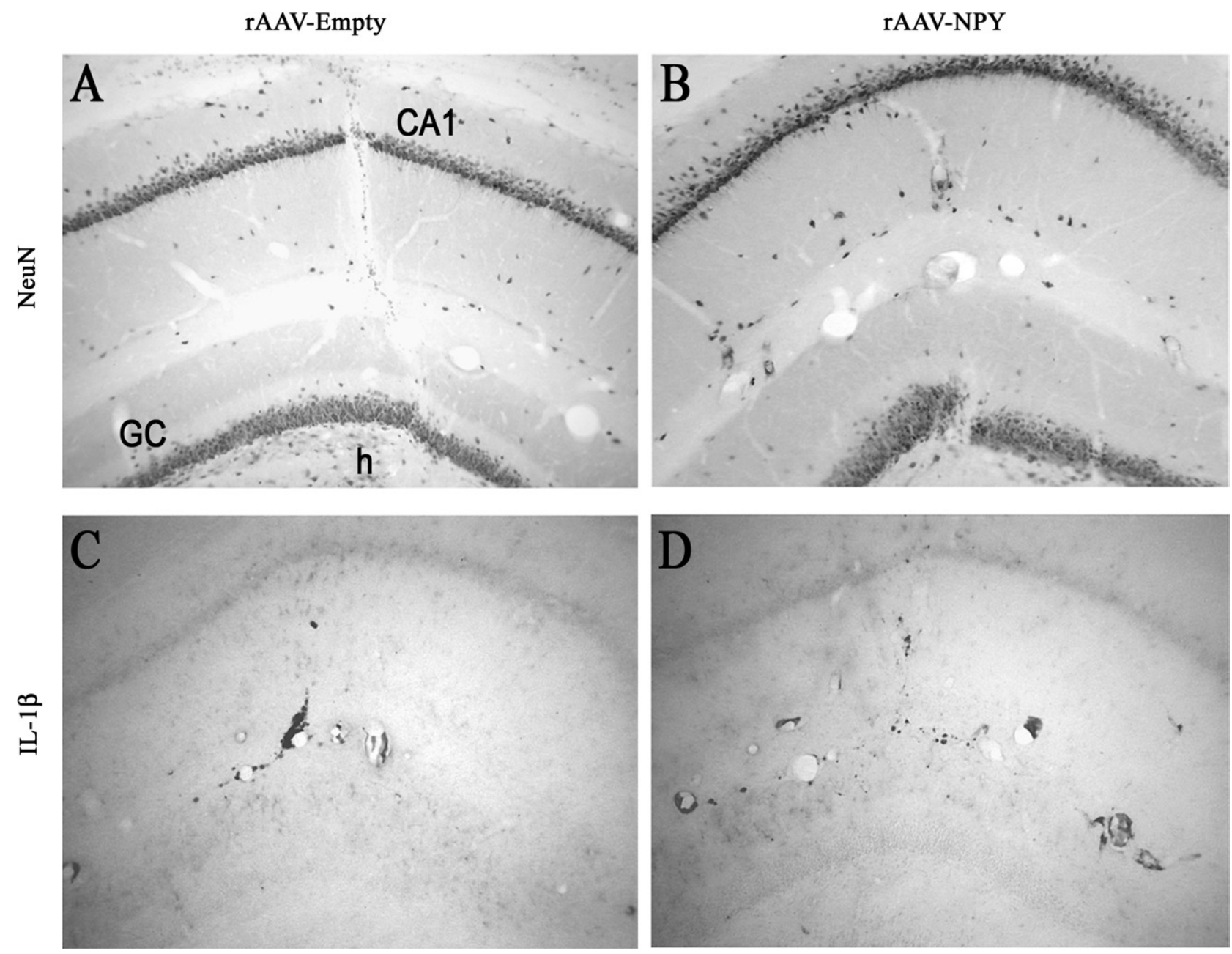

FIG. 2. Neuronal and interleukin (IL)-1 $\beta$ staining in vector injected hippocampus. Photographs show high magnification (20x) of hippocampal sections adjacent to the injection site in representative rats 4 weeks after injection with adeno-associated viral (AAV)1/2-empty (A, C) or AAV1/2-CBA-neuropeptide Y (NPY) (B, D) vector: NeuN staining shows neuron preservation and IL-1 $\beta$ staining depicts lack of inflammation in sections close to the injection needle. CA1 = pyramidal cells; GC = granule cells; $\mathrm{h}=$ hilus. 

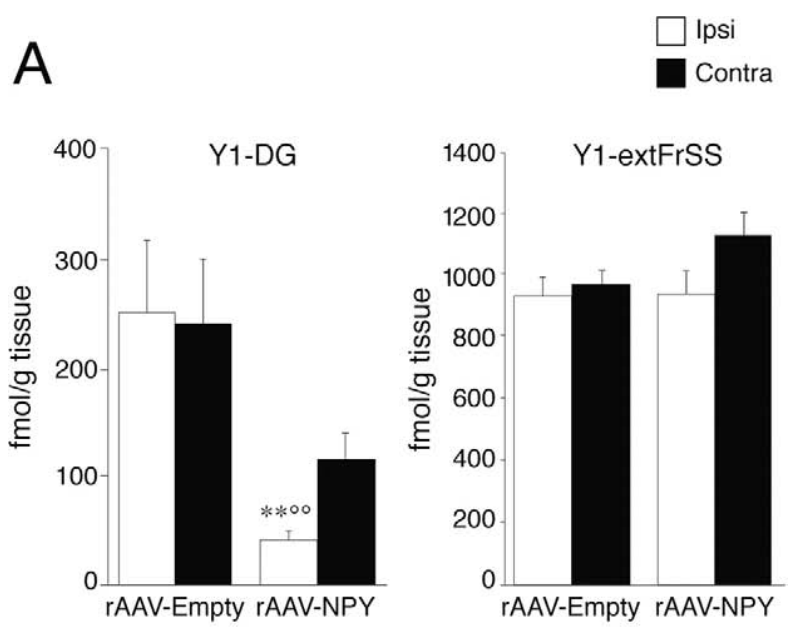

B

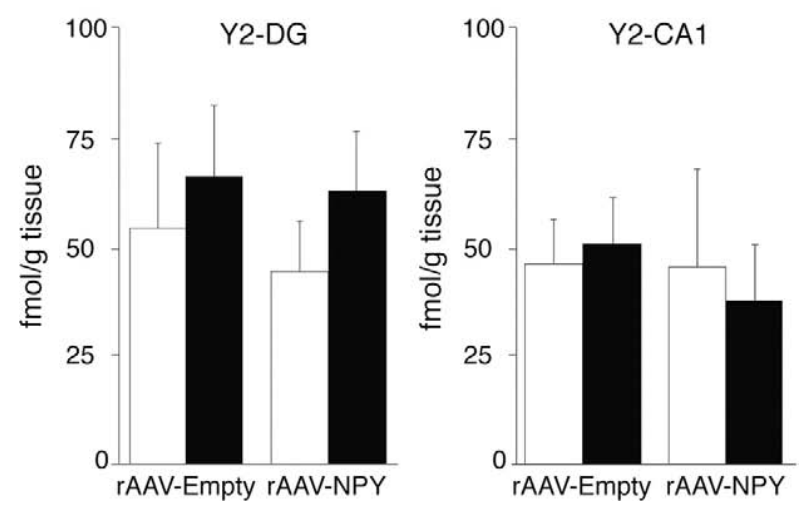

FIG. 3. Neuropeptide $Y$ (NPY)-peptide YY (PYY) receptor binding in rats overexpressing NPY in the hippocampus. NPY-Y1 receptor binding (BIBO-3304 sensitive $\left[{ }^{125} \mathrm{l}\right] \mathrm{Pro}^{34} \mathrm{PYY}$ binding) in the dentate gyrus (DG) of adeno-associated viral (AAV)1/2CBA-NPY injected naïve rats (A): binding to NPY-Y1 receptors was significantly reduced in the DG ipsilateral to the injection site, whereas no changes in binding were detected in brain areas outside the injected hippocampus as exemplified in external frontoparietal cortex/somatosensory area (extFrSS). No significant effects of rAAV-NPY vector injection were found in NPY-Y2 receptor binding (i.e., BIIE-0246 sensitive [ ${ }^{125}$ I]PYY binding) in hippocampal regions (B). Binding was assessed 4 weeks after vector injection in the hippocampus $\left({ }^{\star *} p<0.01\right.$ vs rAAV-empty; ${ }^{\circ 0} p<0.05$ vs contralateral non-injected side by Tukey's test). From Noé et al, 2008. ${ }^{21}$

(FIG. 3). Thus, the Y2 receptors chiefly mediating NPY anticonvulsant activity are preserved in epileptic tissue, whereas Y1 receptors reported to mediate excitatory effects of NPY are down-regulated. ${ }^{13}$ These receptor changes are in accordance with previous evidence showing Y2 receptor upregulation in the hippocampus associated with strong NPY immunoreactivity, ${ }^{13}$ whereas the peptide induces rapid internalization of $\mathrm{Y} 1$ receptors. ${ }^{22}$

AAV-mediated expression of human prepro-NPY under the control of the neuron-specific-enolase promoter reduced EEG seizures induced by intrahippocampal ad- ministration of kainic acid by $50 \%$ when using serotype 2 and by $75 \%$ when using chimeric serotype $1 / 2$ indicating that the higher efficiency and larger spread of gene transduction provided by the chimeric serotype was relevant for effective seizure control. Both vectors significantly delayed the onset time to the first seizures. ${ }^{20}$ When galanin was overexpressed in the hippocampus by serotype 2 AAV vector, an average $40 \%$ decrease in kainate-induced seizures was found, whereas the onset time to seizures did not change,${ }^{10}$ indicating that galanin has less anticonvulsant efficacy as compared to NPY in this model. Chimeric serotype 1/2 AAV-neuron-specificenolase-NPY vector was reported to abolish status epilepticus induced by intraventricular injection of kainic acid in rats. ${ }^{20}$ Finally, this vector also significantly retarded hippocampal kindling acquisition via a selective inhibition of the generalization of seizures, and it significantly increased the threshold current for inducing a local afterdischarge. ${ }^{20,23}$

Subsequent studies ${ }^{11}$ confirmed that AAV-mediated NPY overpression in rat brain could alter seizure sensitivity by injecting the vector in the piriform cortex. Anticonvulsant effects were also shown in this work using AAV vector overexpressing NPY 13-36, a peptide fragment that primarily activates $\mathrm{Y} 2$ receptors.

These experimental findings were obtained by applying gene therapy approaches in normal rat brain with the aim of affecting the onset or the duration of seizures. However, it is clinically relevant to evaluate the antiepileptic efficacy of gene transfer in pathologic chronic epileptic tissue, which is characterized by morphological, molecular, and functional changes that can alter the targets or cellular pathways required for transgene actions. ${ }^{24}$ We recently reported that the intrahippocampal injection of AAV1/2-CBA-NPY in a rat model of chronic epilepsy ensuing from electrically-induced status epilepticus, results in long-lasting (at least 7 weeks) NPY overexpression, induces a decrease of spontaneous seizure frequency, and reduces the symptomatic progression of the disease ${ }^{21}$ (FIG. 4). In this model of epilepsy, spontaneous seizures were not affected by therapeutic doses of phenytoin. ${ }^{25}$ This study provides the proof-ofprinciple evidence that transfer of a gene expressing an endogenous anticonvulsant peptide in epileptogenic brain tissue may offer a new opportunity for therapeutic intervention of epileptic patients. The efficacy of the AAV vector in reducing spontaneous seizures in epileptic rats was highly dependent on the extent of NPY increase in mossy fibers and in fibers of the outer molecular layer of the dentate gyrus and hippocampus proper. Electron microscopic analysis of hippocampal tissue of AAV1/2-CBA-NPY injected rats not exposed to seizures (FIG. 5) showed that NPY immunoreactivity was found in both type 1 (presumably glutamatergic) and type 2 (presumably GABAergic) synapses. This obser- 


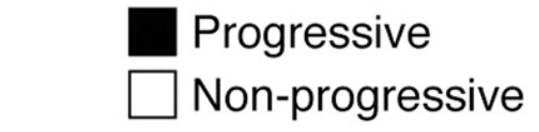

rAAV-Empty

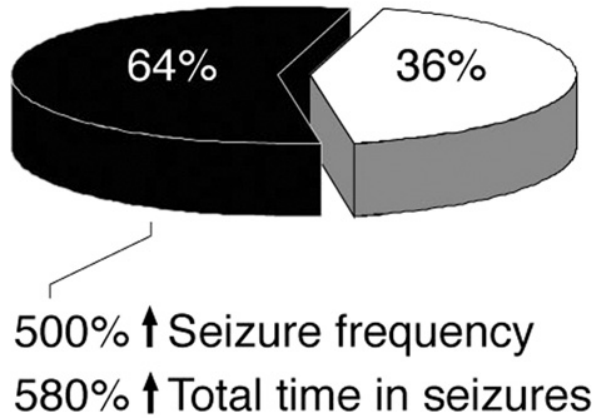

$50 \% \downarrow$ Seizure frequency

$45 \% \downarrow$ Total time in seizures

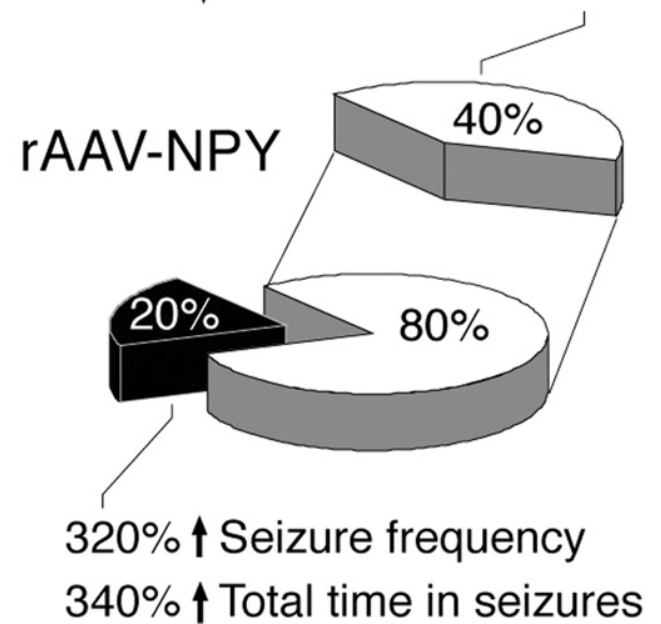

FIG. 4. Effect of rAAV-neuropeptide $Y$ (NPY) on spontaneous seizures in epileptic rats. Proportion of rats with progressive increase in seizure frequency ( $>1.5$-fold increase as compared to the baseline). In the AAV-NPY group, seizure frequency increased $>1.5$-fold only in $20 \%$ of rats as compared to $64 \%$ in the rAAV-empty group ( $p<0.05$, by $\chi^{2}$ test). In the remaining $80 \%$ of rats in the rAAV-NPY group seizure frequency increased less than 1.5-fold as compared to the baseline (nonprogressive). Moreover, half of these rats had a net $50 \%$ decrease in seizure frequency as compared to their respective baseline. Adapted from Noé et al., 2008. ${ }^{21}$

vation is consistent with the immunohistochemical localization of the peptide in granule cell mossy fibers and in hilar interneurons (FIG. 1).

Initial investigations of cognitive functions in naïve rats showed no significant alterations in learning and short-term memory performance after injection of AAV1/2-CBA-NPY, ${ }^{21}$ suggesting that this physiological function is not compromised by the peptide overexpression. Transgene NPY overexpression, provides significant anticonvulsant effects in the kindling mo$\mathrm{del},{ }^{20,23}$ but does not further affect long-term potentiation that is significantly attenuated in kindled rats. ${ }^{23}$

\section{CONCLUSIONS}

Our studies show that intrahippocampal injection of AAV vectors mediating NPY overexpression in rat models of acute seizures, epileptogenesis as well as chronic epilepsy results in long-lasting peptide overexpression in neurons and concomitant powerful anticonvulsant effects. NPY is involved in various functions, including the regulation of blood pressure, circadian rhythms, feeding behavior, anxiety, memory processing, and cognition, ${ }^{26,27}$ raising the possibility of side effects consequent to peptide overexpression. However, many of these functions are mediated by extrahippocampal areas. The highly localized AAV-vector mediated overexpression of NPY makes it unlikely that physiological processes mediated by extrahippocampal brain regions are affected. ${ }^{20,21}$
These findings provide first proof-of-principle evidence that transfer of a gene expressing an endogenous anticonvulsant peptide in chronic epileptic tissue may offer a novel therapeutic intervention, alternative to surgery, in patients with drug-refractory epilepsy.

Further development in gene therapy strategies is now focused particularly in the area of carrier design and its in vivo delivery, cell-type specificity of transgene expression, and transgene expression regulation. Moreover, before treatments can be brought to the clinic, not only the efficacy but also the safety of the treatment, such as the immunogenicity of the transgene product or of the vector serotypes, for example, must be carefully addressed and evaluated using clinically relevant experimental models.

\section{Disclosure}

M.J.D. is a founder and consultant to Neurologix, Inc., a Delaware-based company that has an interest in the commercialization of recombinant AAV vectors for the treatment of neurological disorders.

Acknowledgments: This study was supported by Telethon Onlus Foundation and Fondazione Monzino (AV) and The Academy of Finland and The Juselius Foundation (AP), and Neurologix Inc. We thank Mrs. Merja Lukkari for excellent technical assistance in histology. The authors thank Drs. Valentina Vaghi, Walter Kaufmann, Massimo Rizzi, and Ms. Anna Wieselthaler-Holzl for their contributions to this study. 

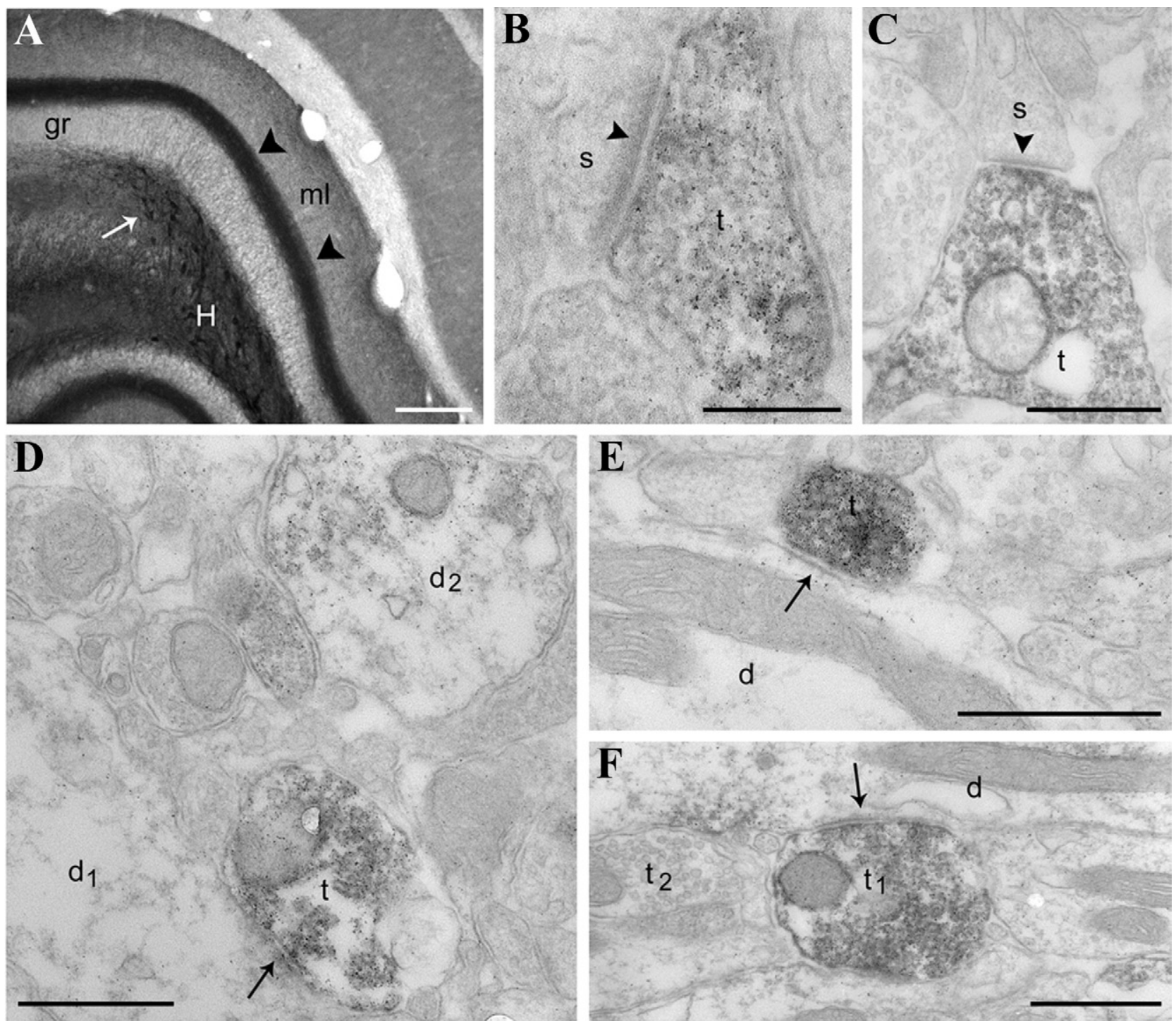

FIG. 5. Light and electron microscopy. A: Digital brightfield photomicrograph of a coronal section of the dentate gyrus from a representative adeno-associated viral (AAV) 1/2-cytomegalovirus-chicken- $\beta$-actin-neuropeptide $Y$ (NPY) injected rat 4 weeks after vector injection. NPY immunoreactivity is present in the somata of hilar $(\mathrm{H})$ interneurones (white arrow), fiber tracts, and in the neuropil of the inner third of the dentate molecular layer ( $\mathrm{ml}$, black arrowheads). B, C: Electron micrographs of immunoperoxidase labeling for NPY in presynaptic axon boutons (t) making type I synaptic contacts (indicated by arrowheads) with dendritic spines (s) in the inner third of the dentate molecular layer. D: Immunoperoxidase labeling of NPY in sporadic dendritic shafts within the dentate molecular layer. Note one dendrite (d2) with peroxidase immunoreaction diffused throughout its cross-section and near an immunolabeled presynaptic bouton (t) forming a type II synapse (indicated by an arrow) with an unlabelled dendrite (d1). Panel E depicts immunoperoxidase labeling of NPY in presynaptic axon boutons (t) making type II synaptic contacts (arrows) with a dendritic shaft (d) in the dentate molecular layer of a rat injected with the rAAV-NPY vector. A representative control (empty-vector injected) rat is depicted in (F). These data indicate that rAAV-mediated overexpression of NPY results in transport of the neuropeptide into both presumed glutamatergic (arrowheads in B and C) and GABAergic (arrows in E) nerve terminals where it undergoes regulated release, but also into dendrites of transfected neurons (d2 in d) where it may be constitutively secreted. Scale bars: $20 \mathrm{~mm}(\mathrm{~A}) ; 0.1 \mu \mathrm{m}(\mathrm{B}) ; 0.2 \mu \mathrm{m}(\mathrm{C}-\mathrm{F})$. gr = granule cell layer; $\mathrm{H}=$ hilus; $\mathrm{ml}=$ molecular layer. From Noé et al., $2008 .{ }^{21}$

\section{REFERENCES}

1. Rogawski MA, Loscher W. The neurobiology of antiepileptic drugs. Nat Rev Neurosci 2004;5:553-564.

2. Foldvary N, Bingaman WE, Wyllie E. Surgical treatment of epilepsy. Neurol Clin 2001;19:491-515.

3. Duncan JS, Sander JW, Sisodiya SM, Walker MC. Adult epilepsy. Lancet 2006;367:1087-1100.

4. Perucca E. Pharmacoresistance in epilepsy: how should it be defined? CNS Drugs 1998;10:171-179.
5. Kaplitt MG, During MJ. Gene therapy in the central nervous system. From bench to bedside, 1st ed. San Diego, CA: Elsevier Inc., 2006.

6. Haberman R, Criswell H, Snowdy S, et al. Therapeutic liabilities of in vivo viral vector tropism: adeno-associated virus vectors, NMDAR1 antisense, and focal seizure sensitivity. Mol Ther 2002; 6:495-500.

7. Raol YH, Lund IV, Bandyopadhyay S, et al. Enhancing GABA(A) receptor alpha 1 subunit levels in hippocampal dentate gyrus inhibits epilepsy development in an animal model of temporal lobe epilepsy. J Neurosci 2006;26:11342-11346. 
8. Mazarati AM. Galanin and galanin receptors in epilepsy. Neuropeptides 2004;38:331-343.

9. Noé F, Nissinen J, Pitkanen A, et al. Gene therapy in epilepsy: the focus on NPY. Peptides 2007;28:377-383.

10. Lin EJ, Richichi C, Young D, Baer K, Vezzani A, During MJ. Recombinant AAV-mediated expression of galanin in rat hippocampus suppresses seizure development. Eur J Neurosci 2003; 18:2087-2092.

11. Foti S, Haberman RP, Samulski RJ, McCown TJ. Adeno-associated virus-mediated expression and constitutive secretion of NPY or NPY13-36 suppresses seizure activity in vivo. Gene Ther 2007; 14:1534-1536.

12. de Lanerolle NC, Kim JH, Robbins RJ, Spencer DD. Hippocampal interneuron loss and plasticity in human temporal lobe epilepsy. Brain Res 1989;495:387-395.

13. Vezzani A, Sperk G, Colmers WF. Neuropeptide Y: emerging evidence for a functional role in seizure modulation. Trends Neurosci 1999;22:25-30

14. Furtinger S, Pirker S, Czech T, Baumgartner C, Ransmayr G, Sperk G. Plasticity of Y1 and Y2 receptors and neuropeptide Y fibers in patients with temporal lobe epilepsy. J Neurosci 2001;21: 5804-5812.

15. Patrylo PR, van den Pol AN, Spencer DD, Williamson A. NPY inhibits glutamatergic excitation in the epileptic human dentate gyrus. J Neurophysiol 1999;82:478-483.

16. Baraban SC, Hollopeter G, Erickson JC, Schwartzkroin PA, Palmiter RD. Knock-out mice reveal a critical antiepileptic role for neuropeptide Y. J Neurosci 1997;17:8927-8936.

17. El Bahh B, Balosso S, Hamilton T, et al. The anti-epileptic actions of neuropeptide $\mathrm{Y}$ in the hippocampus are mediated by $\mathrm{Y} 2$ and not Y5 receptors. Eur J Neurosci 2005;22:1417-1430.
18. Colmers WF, Lukowiak K, Pittman QJ. Neuropeptide Y action in the rat hippocampal slice: site and mechanism of presynaptic inhibition. J Neurosci 1988;8:3827-3837.

19. Greber S, Schwarzer C, Sperk G. Neuropeptide Y inhibits potassium-stimulated glutamate release through Y2 receptors in rat hippocampal slices in vitro. Br J Pharmacol 1994;113:737-740.

20. Richichi C, Lin EJ, Stefanin D, et al. Anticonvulsant and antiepileptogenic effects mediated by adeno-associated virus vector neuropeptide Y expression in the rat hippocampus. J Neurosci 2004; 24:3051-3059.

21. Noé F, Pool AH, Nissinen J, et al. Neuropeptide Y gene therapy decreases chronic spontaneous seizures in a rat model of temporal lobe epilepsy. Brain 2008;131:1506-1515.

22. Pheng LH, Dumont Y, Fournier A, Chabot JG, Beaudet A, Quirion R. Agonist- and antagonist-induced sequestration/internalization of neuropeptide Y Y1 receptors in HEK293 cells. Br J Pharmacol 2003;139:695-704.

23. Sorensen AT, Nikitidou L, Ledri M, et al. Hippocampal NPY gene transfer attenuates seizures without affecting epilepsy-induced impairment of LTP. Exp Neurol 2009;215:328-333.

24. Holmes GL, Ben-Ari Y. Seizing hold of seizures (News and Views). Nat Med 2003;9:994-996.

25. van Vliet EA, van Schaik R, Edelbroek PM, et al. Inhibition of the multidrug transporter P-glycoprotein improves seizure control in phenytoin-treated chronic epileptic rats. Epilepsia 2006;47:672-680.

26. Danger JM, Tonon MC, Jenks BG, et al. Neuropeptide Y: localization in the central nervous system and neuroendocrine functions. Fundam Clin Pharmacol 1990;4:307-340.

27. Hokfelt T, Broberger C, Zhang X, et al. Neuropeptide Y: some viewpoints on a multifaceted peptide in the normal and diseased nervous system. Brain Res Brain Res Rev 1998;26:154-166. 